\title{
Epigenetic activation of WHSC1 functions as an oncogene and is associated with poor prognosis in cervical cancer
}

\author{
ZHUOLIAN YIN ${ }^{1}$, YONG SUN ${ }^{2}$, SUFANG GE $^{1}$ and JUXIANG SUN ${ }^{1}$ \\ Departments of ${ }^{1}$ Obstetrics and Gynecology, and ${ }^{2}$ Reproductive Medicine, \\ Linyi People's Hospital, Linyi, Shandong 276003, P.R. China
}

Received September 25, 2016; Accepted December 2, 2016

DOI: $10.3892 / o r .2017 .5463$

\begin{abstract}
Overexpression of Wolf-Hirschhorn syndrome candidate 1 (WHSC1) is commonly observed in various types of tumors. However, the potential mechanism responsible for this molecular event is poorly understood. In the present study, we found that the mRNA levels of WHSC1 were significantly increased in cervical cancer cells, and that $\mathrm{CpG}$ sites were almost fully methylated in $\mathrm{HaCaT}$ cells, but partially methylated in HeLa and C33A cells. Clinically, the results of quantitative methylation-specific PCR (QMSP) and quantitative real-time PCR (qRT-PCR), showed that methylation levels of the WHSC1 gene were significantly decreased in cervical cancer tumors and inversely correlated with its mRNA expression levels. Both decreased methylation of WHSC1 and increased mRNA were associated with cancer progression and poor prognosis. In addition, overexpression of WHSC1 contributed to cell proliferation, migration and invasion, while cells with WHSC1 knockdown exhibited the opposite effects. AKT/metalloproteinase-2 (MMP-2) signaling was activated and inactivated upon overexpression and silencing of WHSC1, respectively. Silencing of WHSC1 also suppressed tumor growth in a xenograft model. In conclusion, WHSC1 is hypomethylated in cervical cancer, and consequent overexpression of WHSC1 mRNA may promote cervical carcinogenesis by activating the AKT/MMP-2 signaling pathway.
\end{abstract}

\section{Introduction}

Cervical cancer is the second most common type of malignancy in women worldwide, and is the leading cause of cancer-related deaths among women in developing countries (1). Despite improvements in surgery as well as in

Correspondence to: Professor Juxiang Sun, Department of Obstetrics and Gynecology, Linyi People's Hospital, 27 Jiefang Estern Road, Lanshan, Linyi, Shandong 276003, P.R. China E-mail: sunjx1966@163.com

Key words: WHSC1, hypomethylation, survival, proliferation, metastasis, AKT signaling radiotherapy and chemotherapy, the 5-year survival rate of patients with advanced cervical cancer is still unsatisfactory (2). Therefore, understanding the pathogenesis of cervical cancer is beneficial to identifying optimal therapy and determining patient prognosis.

In recent years, accumulating evidence has shown that epigenetic silencing and activation of genes are essential to tumorigenesis and metastasis. For example, epigenetic silencing of ADAMTS19 is commonly involved in colorectal and mucinous ovarian cancer (3). GAS7C, a metastasis suppressor, was found to be hypermethylated in its promoter region in lung cancer (4). Hypomethylation of the ELMO3 promoter induced upregulation of its mRNA and ultimately resulted in the metastasis of lung cancer (5). Aberrant hypomethylation of TKTL1 functions as an oncogene that induces a malignant phenotype of head and neck cancer (6). Therefore, identifying candidate epigenetic targets would be instructive for better understanding of carcinogenesis.

Wolf-Hirschhorn syndrome candidate 1 (WHSC1) is located in the Wolf-Hirschhorn syndrome (WHS) critical region on chromosome 4 p16.3 and is closely related with multiple myeloma (MM) (7). The oncogenic role of WHSC1 in MM was first reported when knockdown of WHSC1 led to cell cycle arrest (8). In addition to regulating the expression of many genes related to the mediation of cell proliferation and adhesion, WHSC1 also regulates the Wnt and NF- $\mathrm{BB}$ signaling pathways in carcinogenesis (9-11). Clinically, two studies have shown that overexpression of WHSC1 occurs in a large variety of tumors and is associated with tumor aggressiveness by analysis of a publicly available gene expression database and conducting experimental analyses, respectively $(12,13)$. All these data suggest that WHSC1 is activated in many types of cancer. However, the biological function and potential mechanism responsible for dysregulated WHSC1 signaling in solid tumors remain unclear.

In the present study, we first identified the epigenetic regulation of WHSC1 in cervical cancer cells and tissues as hypomethylation of its promoter $\mathrm{CpG}$ island. Both methylation and mRNA expression of WHSC1 were significantly correlated with lymph node metastasis and the overall survival of patients. Knockdown of WHSC1 inhibited cell proliferation, migration and invasion in vitro and tumorigenicity in vivo. In addition, the AKT/metalloproteinase-2 (MMP-2) signaling pathway was activated by overexpression of WHSC1. 


\section{Materials and methods}

Clinical samples, cell culture and transfection. All protocols in the present study were approved by the Ethics Committee of Linyi People's Hospital, and written informed consent was obtained from patients before surgery. Primary cervical carcinoma samples $(n=65)$ and their matched non-tumor tissues were collected from patients undergoing surgery at the Department of Obstetrics and Gynecology between February 2009 and July 2011. Non-cancerous tissues were excised at $1.5 \mathrm{~cm}$ from the tumor-free margin. Pathological features were independently evaluated by two pathologists and classified according to the World Health Organization (WHO) classification standard. The clinical data of the patients were extracted from their medical records. Tissue specimens were stored in liquid nitrogen at $-80^{\circ} \mathrm{C}$ until further use.

Human cervical cancer cell lines HeLa, CaSki and C33A were obtained from the Chinese Center for Type Culture Collection (Wuhan, China), and cultured under recommended conditions. HaCaT cells [an immortalized human papillomavirus (HPV)-negative skin keratinocyte line] were used as the normal control. All cells were cultured in a humidified incubator at $37^{\circ} \mathrm{C}$ and $5 \% \mathrm{CO}_{2}$.

The expression of WHSC1 was silenced by siRNA interference with the following sequence: 5'-ACTCGTACACAGC GTGGAGTT-3'. Human WHSC1 cDNA (NM_133330.2) was PCR-amplified, and then subcloned into a lentiviral vector. The siRNA and cDNA were transfected into 293T cells with the packaging plasmids, using Lipofectamine 2000 (Invitrogen, Carlsbad, CA, USA) according to the manufacturer's instructions. The stable WHSC1-silencing and negative control cells were named as siWHSC1 and siRNA, respectively. Stable WHSC1-overexpressing cells and the negative control were named as WHSC1 and vector, respectively.

RNA isolation and quantitative real-time PCR ( $q R T-P C R)$. Total RNA from cell lines and tissue specimens were isolated using TRIzol reagent (Invitrogen). The quantity of RNA was measured using NanoDrop 2000 (Thermo, Waltham, MA, USA). The expression of WHSC1 was determined using qRT-PCR with GAPDH as an internal reference. Isolated mRNA from each sample was transcribed to complementary DNA (cDNA) using a First-Strand cDNA Synthesis kit (Roche, Basel, Switzerland). Primers used for WHSC1 were as follows: 5'-TAGTCATCACAGACACTTCAC-3' (forward) and 5'-TGACTCTCGCTCACAACTCT-3' (reverse); for GAPDH, primers were: 5'-GGAAAGCTGTGGCGTGAT-3' (forward) and 5'-AAGGTGGAAGAATGGGAGTT-3' (reverse). Each sample was analyzed in triplicate and comparative quantification of the target gene was performed based on the cycle threshold $(\mathrm{Ct})$ normalized to GAPDH using the $\Delta \Delta \mathrm{Ct}$ method.

DNA isolation, bisulfite modification and methylation specific-PCR (MSP). Genomic DNA was isolated from cells and frozen tissues using DNeasy Blood and Tissue kit (Qiagen, Duesseldorf, Germany). Bisulfite treatment of genomic DNA $(2 \mu \mathrm{g})$ was conducted using EpiTect Bisulfite kit (Qiagen), and the resulting DNA was used as a template in the MSP assay. The primers of MSP were designed by Methyl Primer Express v1.0 [Applied Biosystems (ABI) Foster City, CA,
USA]. The primers for the methylated sequence of WHSC1 were as follows: 5'-CGAGAGTTTCGGTTTGGTC-3' (forward) and 5'-CTACGATACGTCGCATCCTC-3' (reverse). For the unmethylated sequence, primers were: 5'-GTGAGAG TTTTGGTTTGGTT-3' (forward) and 5'-ACTACAATACAT CACATCCTC-3' (reverse). PCR conditions were 1 cycle at $95^{\circ} \mathrm{C}$ for $15 \mathrm{~min} ; 48$ cycles at $94^{\circ} \mathrm{C}$ for $30 \mathrm{sec}, 56^{\circ} \mathrm{C}$ for $20 \mathrm{sec}$ and $72^{\circ} \mathrm{C}$ for $20 \mathrm{sec}$; and final extension at $72^{\circ} \mathrm{C}$ for $10 \mathrm{~min}$.

Bisulphite genomic sequencing. The primers were designed to amplify the promoter and exon 1 (from -272 to +47 ) for bisulfite sequencing-PCR (BSP) analysis. The following primers used were as follows: 5'-GGATTTGAAAAGTTTGGTT-3' (forward) and 5'-ATCCAACCCAAATACTTCC-3' (reverse). PCR products were purified and then subjected to TA-cloning using pUC18-T vector (Shenggong Biological Engineering Co., Ltd., Shanghai, China). Five clones for each sample were selected for sequencing and analyzed on DNA sequence analyzer (ABI). The methylation data obtained from the sequencing were analyzed using BiQ Analyzer software (Max-Planck Institute, Saarbrücken, Germany) to generate a lollipop diagram.

Quantitative methylation specific-PCR assay. A total of $20 \mathrm{ng}$ of converted DNA was used as a template in the quantitative methylation-specific PCR (QMSP) assay, and the reaction was conducted on a LightCycler 480 (Roche). Leukocyte DNA was methylated with excess SssI methyltransferase (NEB, Beverly, MA, USA) to generate completely methylated DNA, and serial dilutions (50-0.005 ng) of this DNA were used to construct a calibration curve. Each plate contained tissue samples, water blanks and positive controls. Amplifications were performed in 384-well plates, and the reaction $(20 \mu \mathrm{l})$ included $1.2 \mathrm{mmol} / \mathrm{l}$ of primers, $1 \mathrm{U}$ of platinum Taq DNA polymerase, and $200 \mathrm{mmol} / \mathrm{l}$ of each dNTP. The cycling conditions were as follows: $95^{\circ} \mathrm{C}$ for $10 \mathrm{~min}$; 50 cycles of amplification $\left(95^{\circ} \mathrm{C}\right.$ for $30 \mathrm{sec}, 57^{\circ} \mathrm{C}$ for $10 \mathrm{sec}$, and $72^{\circ} \mathrm{C}$ for $20 \mathrm{sec}$ ); and a final step at $72^{\circ} \mathrm{C}$ for $10 \mathrm{~min}$. The relative methylation levels for WHSC1 in each sample were determined as the ratio of QMSP-amplified WHSC1 to ACTB and then multiplied by 100 for easier tabulation.

Demethylation analysis. When cell cultures reached $40 \%$ confluence, HaCaT cells were treated with 0 or $5 \mu \mathrm{M}$ of 5-Aza-2'-deoxycytidine (5-Aza; Sigma-Aldrich, St. Louis, MO, USA) for $72 \mathrm{~h}$. The fresh media containing 5-Aza was changed every $24 \mathrm{~h}$ for 3 days. After $72 \mathrm{~h}$, cells were harvested and subjected to qRT-PCR analysis.

Cell proliferation assay. Cell proliferation was evaluated by absorbance using MTT assay. Briefly, cervical cancer cells (3,000 cells/well) were plated in 96-well plates. At the indicated time-points (24, 48, 72 and $96 \mathrm{~h}$ ), $5 \mathrm{mg} / \mathrm{ml}$ MTT (Sigma-Aldrich) was added to the cells and incubated at $37^{\circ} \mathrm{C}$ for another $4 \mathrm{~h}$. The absorbance at $490 \mathrm{~nm}$ was assessed using an automatic immunosorbent assay reader (BioTek, Winooski, VT, USA).

Wound-healing and Transwell invasion assays. A wound-healing assay was conducted to assess the migratory ability of the cells. Cells were plated onto 6-well plates and 
A

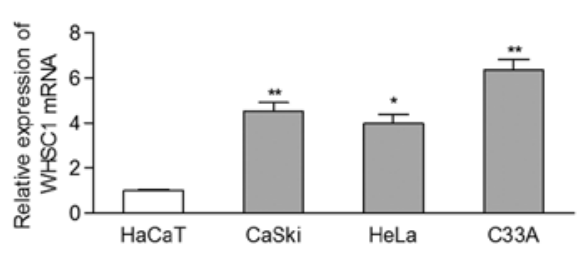

C

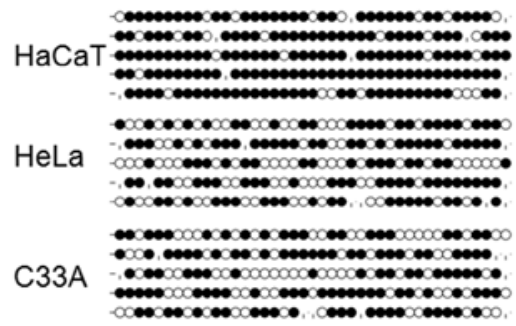

B

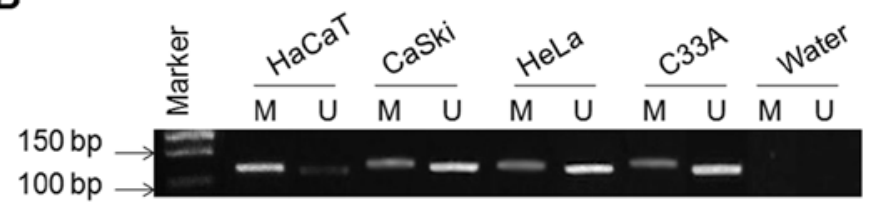

\begin{abstract}
.
\end{abstract}

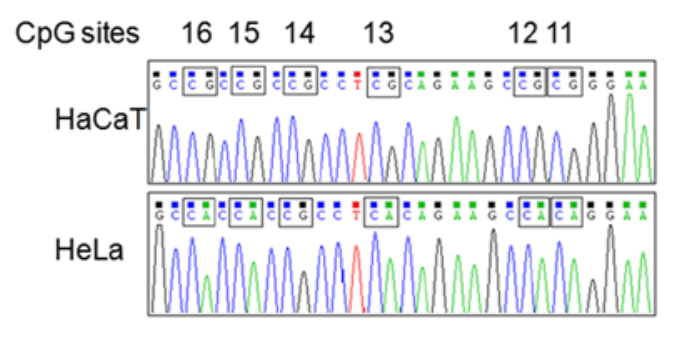

Figure 1. The CpG island of the Wolf-Hirschhorn syndrome candidate 1 (WHSC1) promoter is hypomethylated in cervical cancer. (A) Endogenous WHSC1 levels in cervical cancer cells were determined by qRT-PCR. (B) The methylation status of the WHSC1 promoter region was determined by methylation specific-PCR (MSP); M, methylated; U, unmethylated. (C) Most CpG sites were methylated in the HaCaT cells. Representative sequences of bisulfite sequencing-PCR (BSP) in HeLa and HaCaT cells. Black dots, methylated; white dots, unmethylated. (D) Methylated alleles were assessed using MSP in HaCaT cells after 5-Aza-2'-deoxycytidine (5-Aza) treatment. (E) qRT-PCR analysis of WHSC1 expression in HaCaT cells treated with $5 \mu$ M of 5-Aza for 72 h; ${ }^{*} \mathrm{p}<0.05,{ }^{* *} \mathrm{p}<0.01$.

cultured in complete culture medium until near confluence (80-90\%). The medium was discarded and an artificial wound was made with a $100-\mu 1$ sterile pipette tip. The cells were then washed twice and incubated in serum-free medium for $24 \mathrm{~h}$, after which images were captured.

The BioCoat Matrigel Invasion Chamber (BD Biosciences, Franklin Lakes, NJ, USA) was used to assess cell invasiveness. Cells were resuspended in serum-free medium at a density of $1 \times 10^{6}$ cells $/ \mathrm{ml}$. Cells $\left(1 \times 10^{5}\right)$ were plated into the upper chambers coated with Matrigel. Dulbecco's modified Eagle's medium (DMEM) containing 10\% fetal bovine serum (FBS) was added to the lower chambers. After $24 \mathrm{~h}$, non-migratory cells on the upper surface of the membrane were carefully scraped away. Cells on the lower side of the membrane were fixed, stained with $1 \%$ crystal violet for 2 min, visualized at a magnification of $x 200$ and counted in 6 randomly selected fields. The assays were performed in triplicate.

Tumor xenografts in vivo. A total of $5 \times 10^{6}$ cells transfected with either WHSC1 (siWHSC1) or control siRNA (siRNA) were subcutaneously injected into the right and left flanks, respectively, of male nu/nu mice (6-weeks old). After the tumor growth had been monitored for 5 weeks, the mice were sacrificed, and the tumors were harvested for immunohistochemistry (IHC) analysis. The tumor volume was calculated by the following formula: Width $\mathrm{x}$ length ${ }^{2} \mathrm{x} 0.5$.

Western blot analysis. Proteins were extracted by RIPA lysis buffer, and concentrations were quantified by the BCA protein assay kit (both from Thermo Fisher Scientific). Equal amounts of protein were separated by SDS-polyacrylamide gel electrophoresis (PAGE), and then transferred to polyvinylidine difluoride (PVDF) membranes (Bio-Rad, Hercules, CA, USA). The membranes were blocked and then incubated with the primary antibodies overnight at $4^{\circ} \mathrm{C}$, and then with the horseradish peroxidase-conjugated secondary antibodies.
Bands were visualized using the ECL western blotting detection system (GE Healthcare, Fairfield, CT, USA).

IHC analysis. Xenograft tumors were sliced into $4-\mu \mathrm{m}$ sections and subjected to standard procedures to assess the expression of WHSC1 protein and Ki-67, a proliferation marker. The staining intensity for WHSC1 was scored as 0 (no staining), 1 (weak), 2 (moderate) and 3 (strong), and the staining area was scored as $0(0 \%), 1(1-25 \%), 2(26-50 \%), 3(51-75 \%)$, or $4(76-100 \%)$ on the basis of the percentage of positively stained cells. The sum of the area and the intensity score was calculated to obtain a total score that indicated the expression of the WHSC1 protein. We also used this procedure to assess the protein levels of p-AKT, MMP-2 and MMP-9 in cervical cancer tissues.

Statistical analysis. Data are presented as the mean \pm SD from at least 3 independent experiments. Statistical significance was analyzed using either one-way ANOVA or a two-tail Student's t-test with SPSS 16.0 (IBM, Chicago, IL, USA) and Prism 5 (GraphPad, San Diego, CA, USA). Associations among categorical data were analyzed using a Chi-square or Fisher's exact tests. Overall survival curves were generated by the Kaplan-Meier method and compared using the log-rank test. Cox's proportional hazard regression analysis was used to evaluate independent prognostic factors, the hazard ratio (HR) and the confidence interval (CI). All tests were two-sided, and a p-value of $<0.05$ was considered to indicate a statistically significant result.

\section{Results}

WHSC1 is hypomethylated in cervical cancer cells. First, we evaluated the endogenous mRNA levels of WHSC1 in cervical cancer cells. As shown in Fig. 1A, the mRNA expression of WHSC1 was significantly upregulated in cervical cancer cells 
A
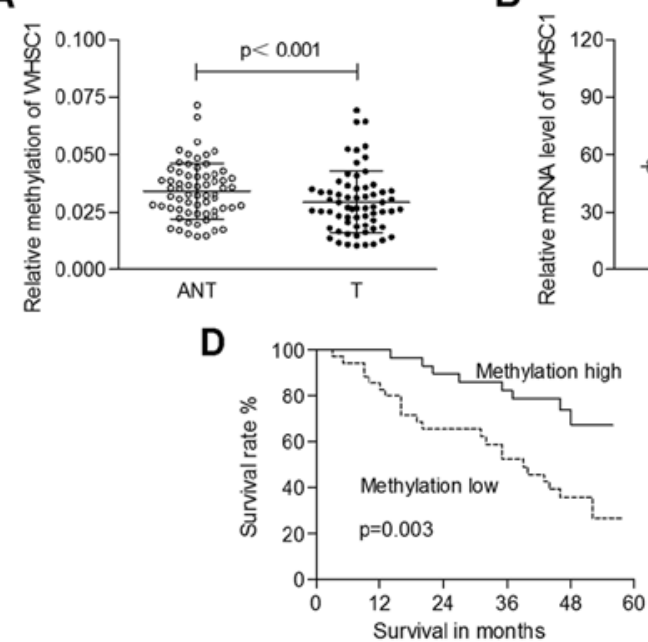

B

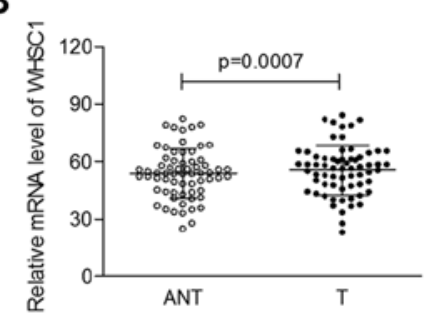

C

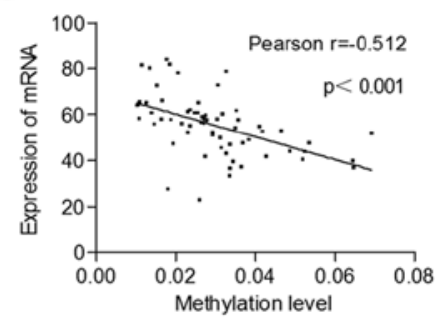

E

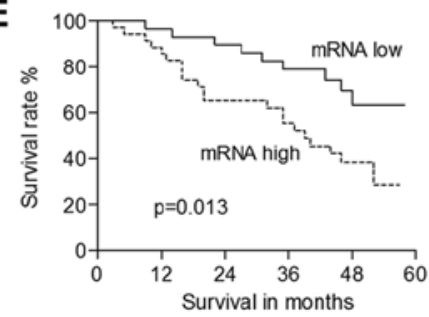

Figure 2. Methylation of the Wolf-Hirschhorn syndrome candidate 1 (WHSC1) gene is negatively correlated with its mRNA levels in 65 cervical cancer cases. (A) Methylation levels of WHSC1 were significantly decreased in tumors compared to adjacent normal tissues. (B) The expression of WHSC1 was upregulated in tumors compared to non-tumor tissues. (C) The expression of WHSC1 was inversely correlated with its methylation status in tumor tissues. (D) Low methylation of the WHSC1 gene in patients indicated a poor prognosis. (E) Increased expression of WHSC1 predicted a poor patient prognosis. The results are expressed as the mean $\pm \mathrm{SD}$.

compared to that in the $\mathrm{HaCaT}$ cells. Then, a $\mathrm{CpG}$ island located in the promoter region of WHSC1 was identified using the UCSC Genome Browser (GRCH38/hg38), suggesting the possibility of epigenetic modifications of the WHSC1 gene. Using MSP analysis, we observed that the WHSC1 promoter was hypomethylated in the CaSki, HeLa and C33A cells, but hypermethylated in the $\mathrm{HaCaT}$ cells (Fig. 1B).

To identify the methylation status of specific $\mathrm{CpG}$ sites, we performed the BSP assay. Our data showed that most CpG sites were methylated in the $\mathrm{HaCaT}$ cells, whereas fewer sites were methylated in the HeLa and C33A cells (Fig. 1C). To further analyze whether hypomethylation of the WHSC1 gene is correlated with its mRNA expression, $\mathrm{HaCaT}$ cells were treated with the DNA methyltransferase inhibitor 5-Aza. The methylation of the allele was decreased in the HaCaT cells treated with $5 \mu \mathrm{M}$ of 5 -Aza in comparison with the control cells (Fig. 1D). In contrast, the expression of WHSC1 was increased in cells that received the 5-Aza treatment (Fig. 1E). These data indicated that hypomethylation of the WHSC1 promoter at least partially regulates its transcriptional activation in cervical cancer.

Upregulation of WHSCl is associated with its hypomethylation in clinical cases. We also ascertained the correlation between the expression of WHSC1 and its promoter methylation status in clinical samples. Genomic DNA extracted from 65 pairs of cervical cancer specimens, and matched normal tissues was amplified by QMSP, and total mRNA was isolated for qRT-PCR analysis. As shown in Fig. 2A, the methylation levels of WHSC1 were significantly decreased in tumors $(0.029 \pm 0.013)$ compared to those in adjacent normal tissues $(0.034 \pm 0.012 ; \mathrm{p}<0.001)$. The mRNA levels of WHSC1 in cancerous tissues $(55.64 \pm 12.97)$ were significantly higher than those in normal tissues (52.76 \pm 12.88 ; $\mathrm{p}=0.0007$; Fig. 2B). Furthermore, we identified a significantly negative correlation between WHSC1 methylation and its mRNA expression (Pearson's correlation, r=-0.512; $\mathrm{p}<0.001$; Fig. 2C).
Dysregulation of WHSCl is correlated with tumor progression and clinical outcomes. The mean value of the methylation levels of WHSC1 in tumors was used as a cut-off to classify cases into 2 groups (low methylation, $\mathrm{n}=35$; high methylation, $\mathrm{n}=30$ ). The hypomethylation of WHSC1 was correlated with higher tumor stage and lymph node metastasis $(\mathrm{p}=0.015$ and 0.004 , respectively; Table I). The mean value of the mRNA levels of WHSC1 in the tumors was used as the threshold to classify patients into 2 groups (low expression, $n=30$; high expression, $\mathrm{n}=35$ ). Upregulation of WHSC1 was significant in cases with lymph node metastasis ( $\mathrm{p}=0.019$; Table I).

The complete clinical follow-up data were analyzed regarding the overall survival rate. We found that both low methylation of WHSC1 and increased mRNA expression were correlated with a poor clinical outcome $(\mathrm{p}=0.003$ and 0.013 , respectively; Fig. 2D and E). Cox proportional hazard regression analysis showed the prognostic significance of the histological type (poor), the International Federation of Gynecology and Obstetrics (FIGO) stage (IIb/IIIa), and lymph node metastasis ( $\mathrm{p}=0.001,0.000$ and 0.000 , respectively; Table II). The low levels of methylation of WHSC1 were associated with a relative risk of death of 3.164 (95\% CI, 1.406-7.120; $\mathrm{p}=0.005$ ), and the high mRNA expression of WHSC1 was correlated with a relative risk of death of 2.589 (95\% CI, 1.183-5.664; $\mathrm{p}=0.017$ ). However, the results of the multivariate analysis revealed that poor differentiation (HR, 3.126; $\mathrm{p}=0.004)$ and high tumor-node-metastasis (TNM) stage $(\mathrm{HR}, 3.787 ; \mathrm{p}=0.001)$ were independently correlated with a significantly increased risk of death (Table II). These data suggested that overexpression of WHSC1 may be involved in cervical carcinogenesis.

Overexpression of WHSC1 promotes cell proliferation, migration and invasion. To ascertain the oncogenic role of WHSC1 in cervical cancer, we performed in vitro experiments to reveal the effects of WHSCl on cell growth and motility. By transducing a vector overexpressing WHSC1, WHSC1 levels were enhanced in the HeLa cells (Fig. 3A). We found that 
Table I. Correlations between clinicopathological characteristics of the cervical cancer patients and WHSC1 methylation or mRNA levels.

\begin{tabular}{|c|c|c|c|c|c|c|c|}
\hline \multirow[b]{2}{*}{ Characteristics } & \multirow{2}{*}{$\begin{array}{c}\text { No. of patients } \\
n=65\end{array}$} & \multicolumn{2}{|c|}{ Methylation level } & \multirow[b]{2}{*}{ P-value } & \multicolumn{2}{|c|}{ Expression of mRNA } & \multirow[b]{2}{*}{ P-value } \\
\hline & & Low, $n=35$ & High, $n=30$ & & Low, $n=30$ & High, $n=35$ & \\
\hline Age (years) & & & & 0.940 & & & 0.679 \\
\hline$<55$ & 30 & 16 & 14 & & 13 & 17 & \\
\hline$\geq 55$ & 35 & 19 & 16 & & 17 & 18 & \\
\hline HPV infection & & & & 0.329 & & & 0.136 \\
\hline No & 24 & 11 & 13 & & 14 & 10 & \\
\hline Yes & 41 & 24 & 17 & & 16 & 25 & \\
\hline Diameter of tumor $(\mathrm{cm})$ & & & & 0.427 & & & 0.427 \\
\hline$<4$ & 29 & 14 & 15 & & 15 & 14 & \\
\hline$\geq 4$ & 36 & 21 & 15 & & 15 & 21 & \\
\hline Histological type & & & & 0.087 & & & 0.263 \\
\hline Well, moderate & 50 & 24 & 26 & & 25 & 25 & \\
\hline Poor & 15 & 11 & 4 & & 5 & 10 & \\
\hline FIGO stage & & & & 0.015 & & & 0.056 \\
\hline $\mathrm{Ib}, \mathrm{IIa}$ & 35 & 14 & 21 & & 20 & 15 & \\
\hline IIb, IIIa & 30 & 21 & 9 & & 10 & 20 & \\
\hline Lymph node metastasis & & & & 0.004 & & & 0.019 \\
\hline No & 31 & 11 & 20 & & 19 & 12 & \\
\hline Yes & 34 & 24 & 10 & & 11 & 23 & \\
\hline
\end{tabular}

WHSC1, Wolf-Hirschhorn syndrome candidate 1; HPV, human papillomavirus; FIGO, International Federation of Gynecology and Obstetrics.

Table II. Clinical characteristics of the cervical cancer patients and the correlatation with overall survival by Cox proportional hazard regression analysis.

\begin{tabular}{|c|c|c|c|c|c|c|}
\hline \multirow[b]{2}{*}{ Parameters } & \multicolumn{3}{|c|}{ Univariate } & \multicolumn{3}{|c|}{ Multivariate } \\
\hline & HR & $95 \% \mathrm{CI}$ & P-value & HR & $95 \% \mathrm{CI}$ & P-value \\
\hline Age, years $(<55 / \geq 55)$ & 1.264 & $0.613-2.607$ & 0.525 & & & \\
\hline HPV infection (no/yes) & 0.747 & $0.362-1.540$ & 0.429 & & & \\
\hline Diameter of tumor $(<4 / \geq 4 \mathrm{~cm})$ & 1.104 & $0.525-2.323$ & 0.794 & & & \\
\hline Histologic type (well, moderate/poor) & 3.642 & $1.629-7.356$ & 0.001 & 3.126 & $1.447-6.753$ & 0.004 \\
\hline FIGO stage (Ib, IIa/IIb, IIIa) & 4.046 & $1.869-8.763$ & 0.000 & 3.787 & $1.731-8.285$ & 0.001 \\
\hline LNM (no/yes) & 4.819 & $2.052-11.317$ & 0.000 & 2.305 & $0.832-6.382$ & 0.108 \\
\hline WHSC1 methylation (high/low) & 3.164 & $1.406-7.120$ & 0.005 & 1.363 & $0.522-3.559$ & 0.527 \\
\hline WHSC1 mRNA level (low/high) & 2.589 & $1.183-5.664$ & 0.017 & 1.607 & $0.721-3.580$ & 0.246 \\
\hline
\end{tabular}

HR, hazard ratio; CI, confidence interval; HPV, human papillomavirus; FIGO, International Federation of Gynecology and Obstetrics; LNM, lymph node metastasis; WHSC1, Wolf-Hirschhorn syndrome candidate 1.

overexpression of WHSC1 contributed to cell proliferation in both the HeLa and C33A cells (Fig. 3B). Upregulation of WHSC1 also enhanced the migratory ability of HeLa and C33A cells based on a wound-healing assay (Fig. 3C). Similarly, the invasive ability of cells was increased by ectopic WHSC1 expression (Fig. 3D). Furthermore, the protein levels of phosphorylated AKT (p-AKT), cyclin D1 (CCND1),
MMP-2 and MMP-9 were increased in cells overexpressing WHSC1 (Fig. 3E). There were no alterations observed regarding the expression of total AKT, irrespective of the presence of WHSC1.

Silencing of WHSC1 inhibits cell growth and motility. We also knocked down WHSC1 in cancer cells by RNA interference 
A

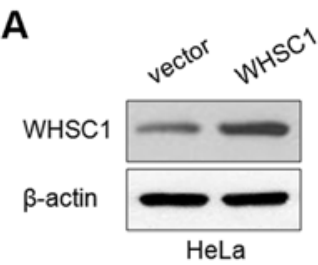

B

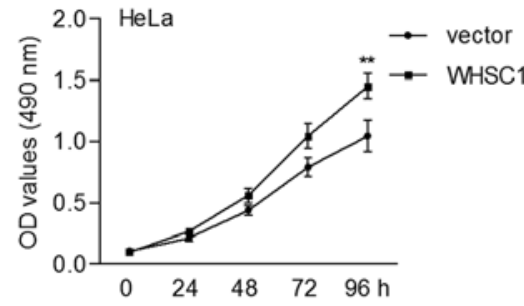

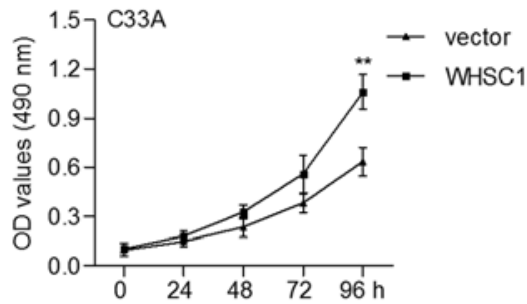
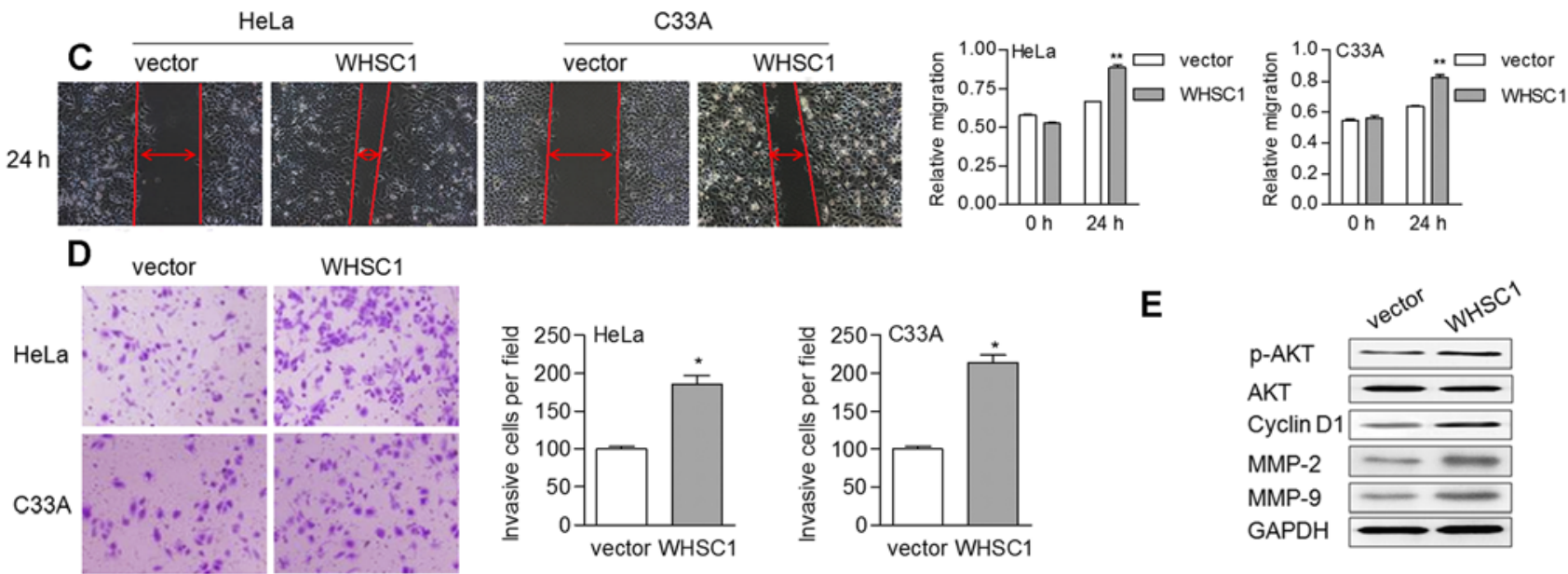

E

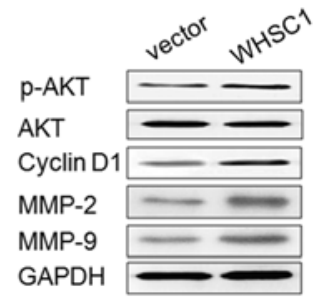

Figure 3. Overexpression of Wolf-Hirschhorn syndrome candidate 1 (WHSC1) promotes cell growth and motility. (A) The expression of WHSC1 after cDNA transfection was determined by western blot analysis. (B) Overexpression of WHSC1 promoted cell proliferation. (C and D) The effects of WHSC1 upregulation on cell migration and invasion were determined using wound-healing and Transwell invasion assays, respectively. (E) Protein levels of p-AKT, cyclin D1 (CCND1), metalloproteinase-2 (MMP-2) and MMP-9 were upregulated after transfection of WHSC1 cDNA. vector, empty vector; WHSC1, cells stably-expressing WHSC1; ${ }^{\mathrm{p}}<0.05,{ }^{* *} \mathrm{p}<0.01$.

A
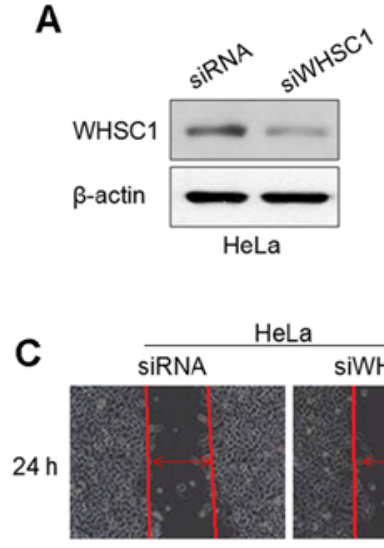

D

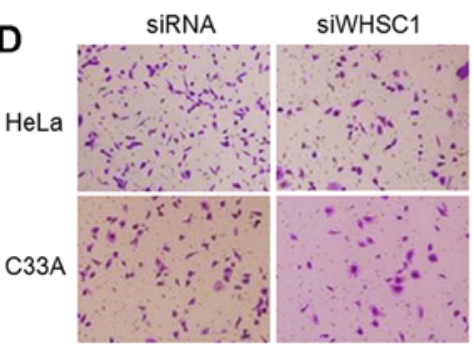

B
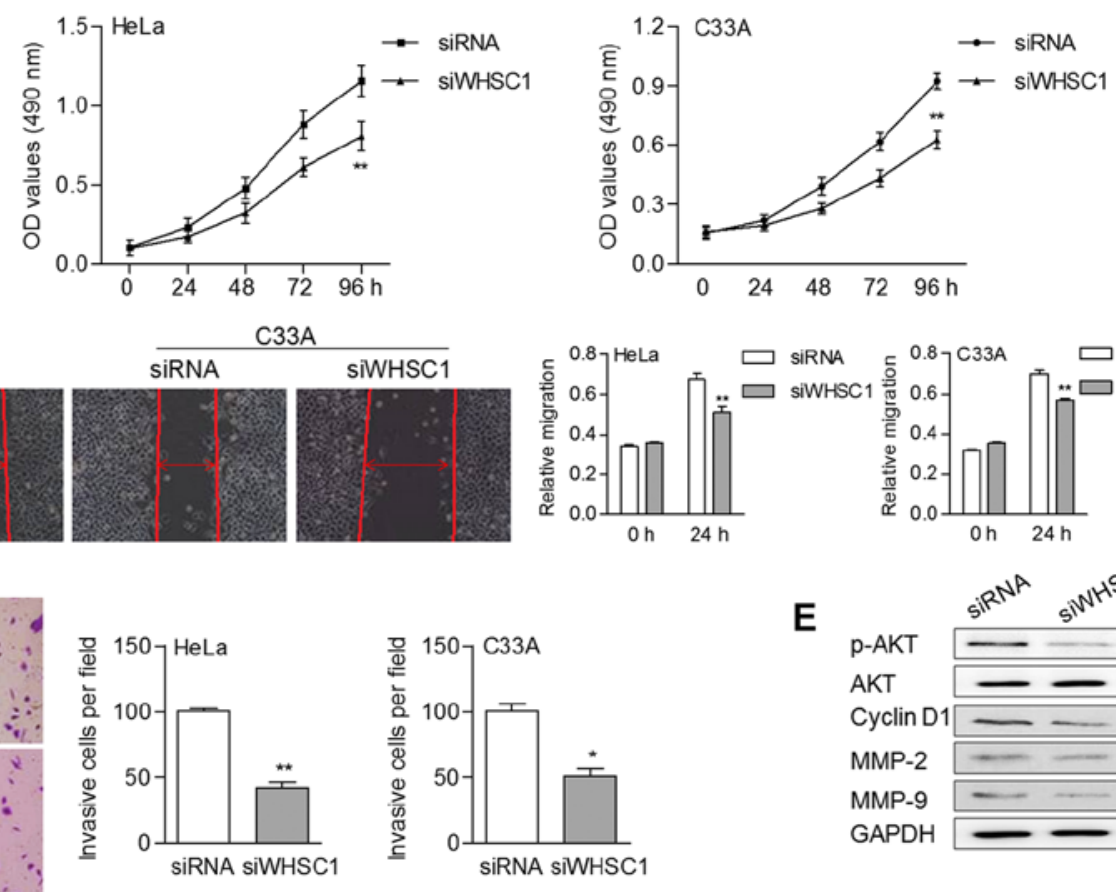
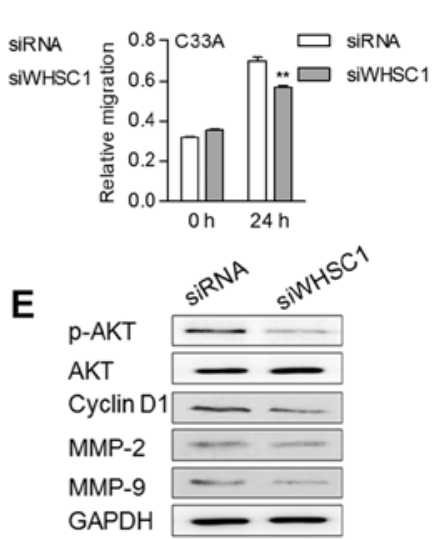

Figure 4. Knockdown of Wolf-Hirschhorn syndrome candidate 1 (WHSC1) attenuates cell growth and motility. (A) Western blotting was used to determine the efficiency of WHSC1 knockdown. (B) The MTT assay was used to evaluate the effect of siWHSC1 on cell growth. (C and D) The migratory and invasive abilities of cells were inhibited by knockdown of WHSC1. (E) Protein levels of p-AKT, cyclin D1 (CCND1), metalloproteinase-2 (MMP-2) and MMP-9 were abated after silencing the expression of WHSC1. siWHSC1, cells transfected with siRNA targeting WHSC1; siRNA, cells transfected with control siRNA; ${ }^{*} \mathrm{p}<0.05 ;{ }^{* *} \mathrm{p}<0.01$.

using siRNA (Fig. 4A). Stable expression of WHSC1 siRNA led to an obvious decrease of cell proliferation (Fig. 4B). In addition, knockdown of WHSC1 suppressed the migratory and invasive abilities of cervical cancer cells (Fig. 4C and D). 
A

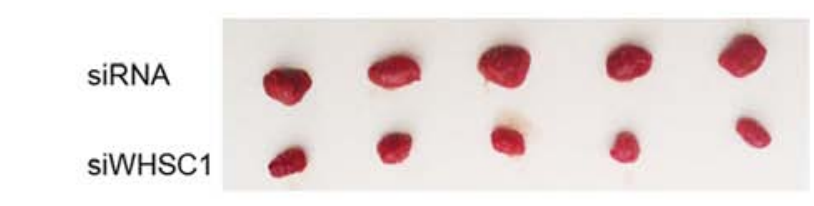

C

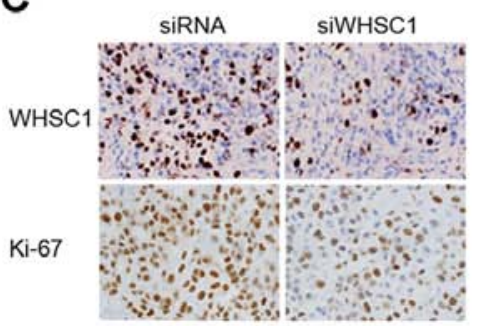

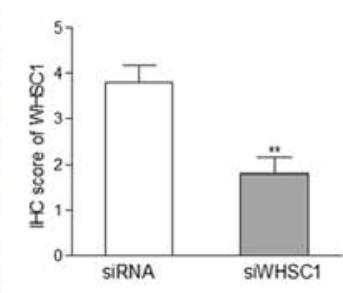

B
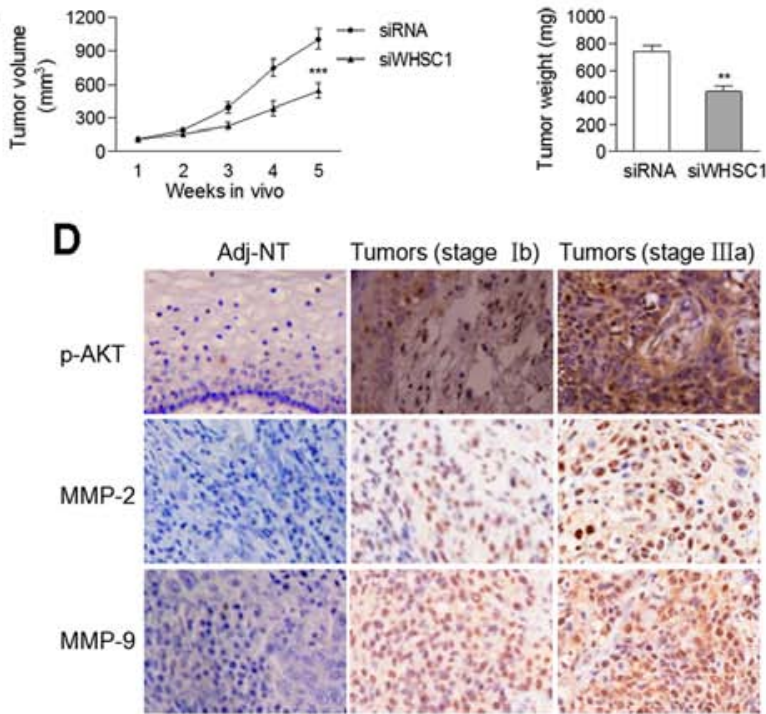

Figure 5. Silencing of Wolf-Hirschhorn syndrome candidate 1 (WHSC1) inhibits tumorigenicity in a xenograft model. (A) A total of 5x10 ${ }^{6} \mathrm{HeLa}$ cells that were stably transfected with siWHSC1 or siRNA were subcutaneously injected into nude mice $(\mathrm{n}=5)$. Mice were sacrificed 35 days after injection, and tumors were excised. Knockdown of WHSC1 led to growth rate inhibition. (B) Tumors comprised of cells with silenced WHSC1 had a lower weight compared with those from the control group. (C) IHC was used to assess the protein levels of WHSC1 and Ki-67 (magnification, x200). The expression of WHSC1 was inhibited in cells stably-expressing siWHSC1. (D) IHC was used to determine the protein levels of p-AKT, metalloproteinase-2 (MMP-2) and MMP-9 in clinical samples. Adj-NT, adjacent non-tumor tissues. ${ }^{* * *} \mathrm{p}<0.01 ;{ }^{* * * *} \mathrm{p}<0.001$.

The protein expression levels of p-AKT, CCND1, MMP-2 and MMP-9 were noticeably decreased in cells with WHSC1 knockdown (Fig. 4E). These data suggested that WHSC1 probably contributed to tumor progression by activating AKT/MMP-2 signaling in cervical cancer.

Knockdown of WHSCl inhibits tumor growth in vivo. Based on the oncogenic effect of WHSC1 observed in the in vitro experiments, a xenograft model of human cervical cancer cells was applied in nude mice. There was a significant decrease in the tumor size and weight of tumors containing cells with WHSC1 knockdown compared to tumors comprised of cells from the control group (Fig. 5A and B). IHC revealed that the protein levels of WHSC1 and $\mathrm{Ki}-67$ were decreased in tumors stably-expressing siWHSC1 (Fig. 5C), suggesting an oncogenic role of WHSC1 in vivo.

WHSC1 is positively correlated with $p-A K T, M M P-2$ and MMP-9 expression in clinical samples. Considering that WHSC1 activated AKT/MMP-2 signaling in vitro, we determined the protein levels of p-AKT, MMP-2 and MMP-9 by IHC in 65 pairs of cervical cancer samples and matched normal tissues. As the degree of malignancy increased, there was a gradual increase in the protein levels of p-AKT, MMP-2 and MMP-9 in clinical samples (Fig. 5D), suggesting that AKT/MMP-2 signaling was activated during cervical tumorigenesis. We found that the mRNA expression of WHSC1 was positively correlated with the protein levels of p-AKT (Spearman $\mathrm{r}=0.292, \mathrm{p}=0.018$ ) and MMP-9 (Spearman $\mathrm{r}=0.304$, $\mathrm{p}=0.014$ ), but not with MMP-2 (Spearman $\mathrm{r}=0.231, \mathrm{p}=0.064$ ).

\section{Discussion}

Epigenetic modification and the consequent dysregulation of genes are emerging as promising novel targets in the treatment and prognostic prediction of cervical cancer $(14,15)$. In the present study, we described a novel mechanism in which epigenetic activation of WHSC1 is induced by hypomethylation of its promoter and provided direct evidence that WHSC1 is a critical oncogene in human cervical cancer.

First, we preliminarily determined the endogenous WHSC1 levels in cervical cancer cells. Our data revealed that WHSC1 is overexpressed in tumors cells, which was similar with findings from previous studies $(13,16)$. Further MSP and BSP analyses ascertained the hypomethylation status of the $\mathrm{CpG}$ island in the promoter region of WHSC1, suggesting that hypomethylation of this $\mathrm{CpG}$ island leads to WHSC1 activation during the development of cervical cancer. Using the publicly available database MethHC (methhc.mbc. nctu.edu.tw), we found that the methylation level of the $\mathrm{CpG}$ island near the WHSC1 promoter was markedly decreased in cervical cancer $(\mathrm{p}<0.005)$ and inversely associated with its mRNA expression (correlation $\mathrm{r}=-0.113, \mathrm{p}=1.11 \times 10^{-16}$; data not shown). In addition, the results from this database showed that the methylation levels of WHSC1 were also decreased in colorectal adenocarcinoma, head and neck squamous, lung squamous and renal clear cell carcinoma. Collectively, all these observations indicated that promoter hypomethylation may be an important mechanism responsible for the activation of WHSC1 in various human cancers. We reviewed previous studies and found that WHSC1 was also reported as a hypomethylated gene in head and neck cancer by genome-wide methylation profiling (17). Our findings provide the direct evidence that hypomethylation of WHSC1 is involved in the pathogenesis of cervical cancer.

Regarding the aforementioned findings, we observed that WHSC1, a specific histone methyltransferase, induced the dimethylation of histone 3 at lysine 36 (H3K36 me2) and decreased the trimethylation of histone 3 at lysine 27 (H3K27 me3) $(18,19)$. WHSC1 regulated the expression of 
TWIST1 and NEK7 through H3K36 me2 (19,20). Presumably, genomic disorders of $\mathrm{H} 3 \mathrm{~K} 36 \mathrm{me} 2$ and tumor-related genes mediated by WHSC 1 may contribute to malignant programming.

We assessed the WHSC1 methylation level in 65 pairs of tumors and corresponding normal tissues, and found that the increased hypomethylation of WHSC1 in tumors indicated tumor progression, suggesting the clinical importance of epigenetic modification. Furthermore, increased expression of WHSC1 was associated with lymph node metastasis and a worse survival rate. Similar observations were also documented in previous studies $(12,13,21)$. By comparing the mRNA levels of WHSC 1 across 3 analyses from Oncomine (www.oncomine.org), we found that the expression of WHSC1 was significantly upregulated in cervical cancer tissues ( $p=9.88 \times 10^{-11}$, data not shown). Our data were consistent with these results, thus supporting the oncogenic role of WHSC1 in the development of cervical tumorigenesis. Upregulated WHSC1 mRNA was not an independent prognostic factor in the present study, while opposing observations were noted in other studies regarding the protein expression of WHSC1 $(22,23)$. Tissue-specific expression and the types of samples assessed may explain these differences. Further analysis based on a larger cohort may provide more accurate information regarding the efficiency of WHSC1 as a prognostic factor.

Finally, the suppressive effect of WHSC1 on cell growth and motility was ascertained, supporting the findings that dysregulated WHSC1 is correlated with lymph node metastasis in clinical cases. In addition, overexpression of WHSC1 led to activation of the AKT signaling pathway and upregulation of MMP-2 and MMP-9, revealing the possible mechanism responsible for the alterations of tumor cell behavior. In prostate cancer, TWIST1 was identified as a target of WHSC1, which induced the migration and invasion of immortalized RWPE-1 cells (19). WHSC1 knockdown resulted in significantly induced apoptosis rates in cells from head and neck squamous cell carcinoma (20). Several tumor-related signaling pathways, such as the Wnt and NF- $\mathrm{KB}$, were also reported to be activated by WHSC1 $(10,11)$. Furthermore, we found that the protein levels of p-AKT and MMP-9 were correlated with WHSC1 in clinical samples, suggesting that the AKT/ MMP-2 signaling pathway is at least partially activated in WHSC1-related cervical carcinogenesis. The matrix metalloproteinases (MMPs) is a large family of proteases known as zinc-containing metalloproteins (24). These enzymes are capable of degrading extracellular matrix proteins (25). MMPs were reported to be critical during advanced stages of tumor progression as regulators of tumor cell migration, invasion and metastasis (26). In addition, both MMP-2 and MMP-9 act as positive regulators of angiogenesis $(27,28)$. Our data expanded the knowledge of the role of WHSC1 in human malignancies, suggesting an oncogenic function of WHSC1 in cervical cancer.

In conclusion, our present findings describe WHSC1 as a novel hypomethylated gene in cervical cancer and provide evidence that this gene is a promising prognostic marker. Furthermore, we suggest that WHSC1 promotes more aggressive types of cervical cancer through the modulation of the AKT/MMP-2 signaling pathway.

\section{References}

1. Siegel R, Naishadham D and Jemal A: Cancer statistics, 2013. CA Cancer J Clin 63: 11-30, 2013.

2. Eskander RN and Tewari KS: Targeting angiogenesis in advanced cervical cancer. Ther Adv Med Oncol 6: 280-292, 2014.

3. Alonso S, González B, Ruiz-Larroya T, Durán Domínguez M, Kato T, Matsunaga A, Suzuki K, Strongin AY, Gimènez-Bonafé $P$ and Perucho M: Epigenetic inactivation of the extracellular matrix metallopeptidase ADAMTS19 gene and the metastatic spread in colorectal cancer. Clin Epigenetics 7: 124, 2015.

4. Tseng RC, Chang JW, Mao JS, Tsai CD, Wu PC, Lin CJ, Lu YL, Liao SY, Cheng HC, Hsu HS, et al: Growth-arrest-specific 7C protein inhibits tumor metastasis via the N-WASP/FAK/F-actin and hnRNP $\mathrm{U} / \beta-\operatorname{TrCP} / \beta$-catenin pathways in lung cancer. Oncotarget 6: 44207-44221, 2015.

5. Søes S, Daugaard IL, Sørensen BS, Carus A, Mattheisen M, Alsner J, Overgaard J, Hager H, Hansen LL and Kristensen LS: Hypomethylation and increased expression of the putative oncogene ELMO3 are associated with lung cancer development and metastases formation. Oncoscience 1: 367-374, 2014.

6. Sun W, Liu Y, Glazer CA, Shao C, Bhan S, Demokan S, Zhao M, Rudek MA, Ha PK and Califano JA: TKTL1 is activated by promoter hypomethylation and contributes to head and neck squamous cell carcinoma carcinogenesis through increased aerobic glycolysis and HIF1alpha stabilization. Clin Cancer Res 16: 857-866, 2010.

7. Chesi M, Nardini E, Lim RS, Smith KD, Kuehl WM and Bergsagel PL: The $t(4 ; 14)$ translocation in myeloma dysregulates both FGFR3 and a novel gene, MMSET, resulting in IgH/MMSET hybrid transcripts. Blood 92: 3025-3034, 1998.

8. Lauring J, Abukhdeir AM, Konishi H, Garay JP, Gustin JP, Wang Q, Arceci RJ, Matsui W and Park BH: The multiple myeloma associated MMSET gene contributes to cellular adhesion, clonogenic growth, and tumorigenicity. Blood 111: 856-864, 2008.

9. Brito JL, Walker B, Jenner M, Dickens NJ, Brown NJ, Ross FM, Avramidou A, Irving JA, Gonzalez D, Davies FE, et al: $M M S E T$ deregulation affects cell cycle progression and adhesion regulons in $\mathrm{t}(4 ; 14)$ myeloma plasma cells. Haematologica 94 : 78-86, 2009.

10. Toyokawa G, Cho HS, Masuda K, Yamane Y, Yoshimatsu M, Hayami S, Takawa M, Iwai Y, Daigo Y, Tsuchiya E, et al: Histone lysine methyltransferase Wolf-Hirschhorn syndrome candidate 1 is involved in human carcinogenesis through regulation of the Wnt pathway. Neoplasia 13: 887-898, 2011.

11. Yang P, Guo L, Duan ZJ, Tepper CG, Xue L, Chen X, Kung HJ, Gao AC, Zou JX and Chen HW: Histone methyltransferase NSD2/MMSET mediates constitutive NF- $\kappa \mathrm{B}$ signaling for cancer cell proliferation, survival, and tumor growth via a feed-forward loop. Mol Cell Biol 32: 3121-3131, 2012.

12. Kassambara A, Klein B and Moreaux J: MMSET is overexpressed in cancers: Link with tumor aggressiveness. Biochem Biophys Res Commun 379: 840-845, 2009.

13. Hudlebusch HR, Santoni-Rugiu E, Simon R, Ralfkiær E, Rossing HH, Johansen JV, Jørgensen M, Sauter G and Helin K: The histone methyltransferase and putative oncoprotein MMSET is overexpressed in a large variety of human tumors. Clin Cancer Res 17: 2919-2933, 2011.

14. Yin A, Zhang Q, Kong X, Jia L, Yang Z, Meng L, Li L, Wang X, Qiao Y, Lu N, et al: JAM3 methylation status as a biomarker for diagnosis of preneoplastic and neoplastic lesions of the cervix. Oncotarget 6: 44373-44387, 2015.

15. Sood S, Patel FD, Ghosh S, Arora A, Dhaliwal LK and Srinivasan R: Epigenetic alteration by DNA methylation of ESRI, $M Y O D 1$ and $h T E R T$ gene promoters is useful for prediction of response in patients of locally advanced invasive cervical carcinoma treated by chemoradiation. Clin Oncol 27: 720-727, 2015.

16. Gu C, Feng L, Peng H, Yang H, Feng Z and Yang Y: MTDH is an oncogene in multiple myeloma, which is suppressed by Bortezomib treatment. Oncotarget 7: 4559-4569, 2016.

17. Zhang XY, Li M, Sun K, Chen XJ, Meng J, Wu L, Zhang P, Tong $X$ and Jiang WW: Decreased expression of GRIM-19 by DNA hypermethylation promotes aerobic glycolysis and cell proliferation in head and neck squamous cell carcinoma. Oncotarget 6: 101-115, 2015. 
18. Popovic R, Martinez-Garcia E, Giannopoulou EG, Zhang Q, Zhang Q, Ezponda T, Shah MY, Zheng Y, Will CM, Small EC, et al: Histone methyltransferase MMSET/NSD2 alters EZH2 binding and reprograms the myeloma epigenome through global and focal changes in H3K 36 and H3K 27 methylation. PLoS Genet 10: e1004566, 2014.

19. Ezponda T, Popovic R, Shah MY, Martinez-Garcia E, Zheng Y, Min DJ, Will C, Neri A, Kelleher NL, Yu J, et al: The histone methyltransferase MMSET/WHSC1 activates TWIST1 to promote an epithelial-mesenchymal transition and invasive properties of prostate cancer. Oncogene 32: 2882-2890, 2013.

20. Saloura V, Cho HS, Kiyotani K, Alachkar H, Zuo Z, Nakakido M, Tsunoda T, Seiwert T, Lingen M, Licht J, et al: WHSC1 promotes oncogenesis through regulation of NIMA-related kinase-7 in squamous cell carcinoma of the head and neck. Mol Cancer Res 13: 293-304, 2015.

21. Yang S, Zhang Y, Meng F, Liu Y, Xia B, Xiao M, Xu Y, Ning X, $\mathrm{Li} \mathrm{H}$ and Lou G: Overexpression of multiple myeloma SET domain (MMSET) is associated with advanced tumor aggressiveness and poor prognosis in serous ovarian carcinoma. Biomarkers 18: 257-263, 2013.

22. Zhou P, Wu LL, Wu KM, Jiang W, Li JD, Zhou LD, Li XY, Chang S, Huang Y, Tan $\mathrm{H}$, et al: Overexpression of MMSET is correlation with poor prognosis in hepatocellular carcinoma. Pathol Oncol Res 19: 303-309, 2013.
23. Xiao M, Yang S, Chen J, Ning X, Guo L, Huang K and Sui L: Overexpression of MMSET in endometrial cancer: A clinicopathologic study. J Surg Oncol 107: 428-432, 2013.

24. Hrabeta J, Eckschlager T, Stiborova M, Heger Z, Krizkova S and Adam V: Zinc and zinc-containing biomolecules in childhood brain tumors. J Mol Med (Berl) 94: 1199-1215, 2016.

25. Basset P, Bellocq JP, Wolf C, Stoll I, Hutin P, Limacher JM, Podhajcer OL, Chenard MP, Rio MC and Chambon P: A novel metalloproteinase gene specifically expressed in stromal cells of breast carcinomas. Nature 348: 699-704, 1990.

26. Chabottaux V and Noel A: Breast cancer progression: Insights into multifaceted matrix metalloproteinases. Clin Exp Metastasis 24: 647-656, 2007.

27. Nelson AR, Fingleton B, Rothenberg ML and Matrisian LM Matrix metalloproteinases: Biologic activity and clinical implications. J Clin Oncol 18: 1135-1149, 2000.

28. Raithatha SA, Muzik H, Muzik H, Rewcastle NB, Johnston RN, Edwards DR and Forsyth PA: Localization of gelatinase-A and gelatinase-B mRNA and protein in human gliomas. Neuro Oncol 2: 145-150, 2000 